In Amsterdam hat man sich entschlossen, in Zukunft alle Fäcalwässer auf schwefelsaures Ammoniak $z u$ verarbeiten und ist man im Augenblick mit dem Bau einer Einrichtung beschäftigt, mittels welcher täglich $250 \mathrm{cbm}$ Fäcalwasser in 24 Stunden verarbeitet werden kann. Diese neue Einrichtung soll am 1. October 1891 in Betrieb gesetzt werden.

Amsterdam, März 1891.

\section{Quantitative Bestimmung der löslichen Chloride, Bromide und Jodide.}

$$
\text { Von }
$$

\section{Prof. Dr. L. L. de Koninck}

und Dr. Ed. Nihoul,

Assistent an der Universität in Lüttich.

H. Quantin veröffentlichte i. J. 1886 ${ }^{1}$ ) eive durch seine Einzelnheiten neue Methode für die Bestimmung der Schwefelsäure und namentlich der schwefelsauren Alkalien, die gestützt ist auf die Reaction zwischen diesen Salzen und dem Baryumchromate. Unter gewissen bestimmten Bedingungen erbält man: $\mathrm{M}_{2} \mathrm{SO}_{4}+x \mathrm{BaCrO}_{4}=\mathrm{Ba} \mathrm{SO}_{4}+(\mathrm{x}-1) \mathrm{BaCrO}_{4}$

$$
+\mathrm{M}_{2} \mathrm{CrO}_{4}
$$

$M$ vertritt hier ein monovalentes Metall. Man beseitigt das Baryumsulfat und den Überschuss an Chromat desselben Metalls und bestimmt dann das gebildete lösliche Chromat auf volumetrischem Wege.

$\mathrm{Da}$ die Menge des letzteren sich proportional zu der der Schwefelsäure verhält, kann man das Gewicht der Schwefelsäure, welche sich in der Probe befindet, berechnen. Unserer Meinung nach könnte eine ähnliche Methode für Chlormetalle anwendbar sein ${ }^{2}$ ).

Man weiss ja (die Reaction wird in der volumetrischen Bestimmung der Chlormetalle durch Silbernitrat, um das Ende der Reaction festzustellen, angewandt), dass das Silberchromat mit den Chloralkalien schnell reagirt, um Silberchlorid und ein lösliches Chromat zu bilden.

$2 \mathrm{M} \mathrm{Cl}+\mathrm{Ag}_{2} \mathrm{CrO}_{4}=2 \mathrm{Ag} \mathrm{Cl}+\mathrm{M}_{2} \mathrm{CrO}_{4}$

Wenn man also ein neutrales Chlormetall mit Silberchromat im Überschuss behandelt, so wird man folgende Reaction erbalten $2 \mathrm{M} \mathrm{Cl}+\mathrm{x} \mathrm{Ag}_{2} \mathrm{CrO}_{4}=2 \mathrm{AgCl}+(\mathrm{x}-1) \mathrm{Ag}_{2} \mathrm{CrO}_{4}$

1) Monit. scient. Quesneville 16, 1222 .

2) Unsere Methode bietet auch einige Ähnlichkeiten mit der von Bohlig dar, welche gestützt ist auf die Reaction zwischen den Chlormetallen und Silberoxalat, sowie auf die Bestimmung des gebildeten löslichen Oxalats durch Chamăleon. (Z. anal. Ch. 1885, 408.)
Da sich die Menge des gebildeten löslichen Chromats zu der der Chlormetalle molecular proportional rerbält, so bleibt nur, wie in der erwähnten Methode von Quantin, durch völlige oder theilweise Filtrirung den Überschuss an unlöslichem Chromat zu entfernen und das in der Flüssigkeit enthaltene Chromat zu bestimmen, um endlich durch Berechnung das zu bestimmende Chlormetall zu erhalten. Quantin schlug vor, das Chromat durch das Penny'sche Verfahren vermittels des schwefelsauren Eisenoxyduls zu bestimmen und das Ende der Reaction durch Tüpfelprobe mit Ferricyankalium zu erkennen.

Andrews und Lancelot ${ }^{3}$ ), welche ebenfalls vorschlugen, das Baryumchromat für die Bestimmung der Schwefelsäure anzuwenden, titriren das gebildete lösliche Chromat durch die K. Zulkowski'sche ${ }^{4}$, von Crismer ${ }^{5}$ ) verbesserte Methode, d. i. mit unterschwefligsaurem Natron vermittels Jodkalium und in Gegenwart von Schwefelsäure, was ja genauer und besonders leichter ist, als mit schwefelsaurem Kisenoxydul. Deshalb haben wir diesem letzten Verfabren den Vorzug gegeben.

Damit diese Methode richtige Resultate geben kann, muss man reines Silberchromat verwenden, welches ron jedem anderen Chromat und jeder mit dem Chlormetalie reagirfähigen Silberverbindung frei ist. Die Auflösung muss ferner von jedem Metall frei sein, welches ein unlösliches Chromat bilden könnte, z. B. Baryum oder Blei. Man muss ferner unter solchen Bedingungen arbeiten, dass das im Überschuss angewendete Silberchromat völlig unaufgelöst bleibt.

Wir waren demnach gezwungen, vor Allem die Darstellungsmethode des Silberchromats festzustellen; wir mussten ferner die Löslichkeit des Products nicht allein in Wasser und in den verdünnten Säuren bestimmen, sondern auch in den chromsauren Alkalien und in den Salzen, die gewöhnlich mit den Chlormetallen vorkommen. Dann nur wurde es uns möglich, zu suchen, $o b$ die im Princip richtige Methode fähig wäre in der Praxis befriedigende Resultate zu geben.

1. Darstellung des Silberchromats. Man wiegt Silbernitrat und rein neutrales Kaliumchromat im Molecularverhältnisse

$$
\left.2 \mathrm{Ag} \mathrm{NO} \mathrm{K}_{3}: \mathrm{K}_{2} \mathrm{CrO}_{4}=339,1: 194,35^{6}\right)
$$

3) American Chem. J. 1889, XI, No. 8.

4) J. f. pract. Ch. 1868 Bd. 103, 351.

5) Ber. deutsch. ch. G. 1884, 642.

6) $\mathrm{Cr}=52,45$. Nach der neueren Arbeit von C. Meineke (Liebig's Ann. 261, 339), welche nach der Redaction der unserigen erschien, ist das Atomgewicht des Chroms 51,94, folglich $\mathrm{Ag}_{2} \mathrm{CrO}_{4}$ $=193,84$ 
ab, d. h. und $7 \mathrm{Th}$. Silbernitrat auf $4 \mathrm{Th}$. Chromat. Man löst jedes für sich auf und giesst beide Auflösungen zusammen; das gefällte Silberchromat wird durch Decantation gewaschen und unter Wasser im Dunkeln aufbewahrt. Für diese Darstellung darf man das Kaliumdichromat nicht anwenden.

2. Eigenschaften des Silberchromats. Die Genaujgkeit unseres Verfahrens und sogar die Möglichkeit, dasselbe für die Bestimmung kleinerer Mengen anzuwenden, hängt selbstverständlich von der Cöslichkeit des Silberchromats ab.

Die Litteratur enthält unseres Wissens keine genaue diesbezügliche Angabe. Die einzigen Auskünfte, die wir darüber ausfindig machen konnten, sind folgende:

Nach B. Biscaro? ) würde die Anwesenheit von Nitraten und wahrscheinlich auch von Sulfaten die Bildung von Silberchromat bei Anwendung von Kaliumchromat als Indicator in der Bestimmung der Chlormetalle verzögern.

R. F. Carpente ${ }^{8}$ ), der die von Biscaro erhobene Frage quantitativ verfolgt hat, gibt einige Zahlen an, die für unsere Sache unwichtig sind. H. Kämmerer ${ }^{9}$ ) sagt, noch immer bei Gelegenheit der Chlorbestimmung durch das Mohr'sche Verfahren, dass das Silberchromat in Wasser löslich, aber in Kaliumchromat unlöslich ist; er gibt keine Zahlen als Belege. Mohr ${ }^{10}$ ) sagt bei Besprechung des Silberchromats: ,ein in Wasser fast unlösliches Salz".

Das Ungenügende an diesen Auskünften hat uns zu einigen Versuchen Veranlassung gegeben.

Löslichkeit des Silberchromats in reinem Wasser. Reines Silbercbromat wurde mit destillirtem Wasser mehrere Stunden bei gewöhnlicher Temperatur (etwa 17 ${ }^{\circ}$ ) öfters geschüttelt, die Flüssigkeit färbte sich hellgelb. $1000 \mathrm{~g}$ dieser Lösung, auf dem Wasserbade eingedampft, ergaben einen geringen, rothbraunen Rückstand, dessen Gewicht nach dem Trocknen bei $100^{\circ}$ war.

$$
\begin{array}{ll}
\text { 1. Versuch } & 36,5 \mathrm{mg} \\
2 . & 39,4
\end{array}
$$

Mittel: $37,9 \mathrm{mg}$. Die Analyse von $34,3 \mathrm{mg}$ desselben Rückstandes ergab:

$$
\begin{array}{lr}
\mathrm{Ag}_{2} \mathrm{O} & 9,97 \mathrm{mg} \\
\mathrm{CrO}_{3} & 24,28 .
\end{array}
$$

Nach diesen Resultaten ist der Verdunstungsrückstand also wirklich neutrales Silberchromat und kein Dichromat, welches man unter Wirkung des Wassers aus dem neutralen Chromat gebildet, hätte vermuthen können.

7) Ann. Chim. med. farm. [4] 1, 241, in Jahresb. von Liebig 1885, 1899.

$\left.{ }^{8}\right)$ Chem. Soc. Ind. 5, 286 in Jbr. f. 1886, 1898.

$\left.{ }^{9}\right)$ Rep. d. anal. Chem. 1885, 398.

10) Mohr's Lehrb. d. Titrirmeth. 6. Aufl. bearb. v. Classen, S.399.
Nach dem Mittelwerth dieser beiden $\mathrm{Be}-$ stimmungen ist also das Silberchromat in 26378 Th. kaltem Wasser löslich. Die Wärme begünstigt die Löslicbkeit, denn als wir destillirtes Wasser mit Silberchromat auf dem Wasserbade während 2 Stunden auf $70^{\circ}$ erwärmten, erzielten wir eine dunkler gefärbte Auflösung als mit kaltem Wasser, was schon eine grössere Löslichkeit des Silberchromats angibt. $1000 \mathrm{~g}$ dieser Auflösung ergaben $109,7 \mathrm{mg}$ neutrales Silberchromat. Dieses löst sich also bei $70^{\circ}$ in $9116 \mathrm{Th}$. Wasser auf. Es geht daraus hervor, dass die Löslichkeit bei $70^{\circ}$ nahe an dreimal stärker ist, als bei der gewöhnlichen Temperatur ${ }^{11}$ ).

Löslichkeit in Kaliumchromaten. Das Silberchromat ist in den sehr concentrirten Auflösungen von Kaliumchromat und namentlich Kaliumdichromat ein wenig löslich. Für verdünnte Auflösungen ist es nicht der Fall; im Gegentheil, wenn man der wässerigen Auflösung des Silberchromats einige Tropfen einer Kaliumchromatlösung hinzufügt, so wird die Flüssigkeit trübe und das gelöste Silberchromat wird gefällt.

Wir wollen vorübergehend bemerken, dass die Zufügung von Silbernitrat zu dieser Auflösung auch die Fällung des Silberchromats zur Folge hat. Diese Thatsachen erinnern an das, was bei der Silberprobe nach Gay-Lussac am Ende der Operation vorkommt.

Löslichkeit in verdünnter Salpetersäure. Das Silberchromat löst sich bei gewöhnlicher Temperatur in:

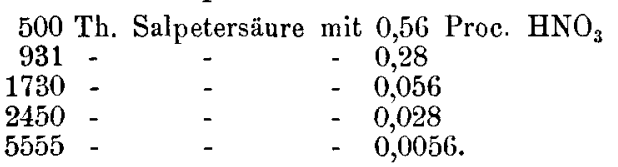

Wir haben ferner festgestellt, dass das Vorbandensein von Natriumacetat diese Löslichkeit nur in geringem Verbältnisse verringert, oder besser gesagt, dass die Löslichkeit in Essigsäure nicht viel geringer ist als in Salpetersäure; $1 \mathrm{Th}$. des Silberchromats ist bei Anwesenbeit von 1 Proc. Natriumacetat löslich in:

$725 \mathrm{Th}$. Salpetersäure mit 0,56 Proc. $\mathrm{HNO}_{3}$

$$
2222-0,056 .
$$

Das Kaliumchromat wie Natriumacetat fällt das Silberchromat aus seinen Auflösungen in Salpetersäure nur unvollständig. Anders verhält sich die Sache bei den Auflösungen des Silberchromats in Nitraten. Aus solchen wird durch eine verdünnte Auf-

11) Daraus kann man schliessen, dass die titrimetrische Bestimmung der Chloride durch Mohr's Verfahren in der Kälte stattzufinden habe. 
lösung von Kaliumchromat das Silberchromat vollständig gefällt.

Wir haben bei Gelegenheit dieser verschiedenen Versuche die Empfindlichkeit der Reaction zwischen dem Kaliumchromat und Silbernitrat untersucht. Giesst man in 200 cc Wasser zugleich cubikcentimeterweise eine $\frac{N}{100}$ Lösung eines jeden dieser Salze bei gewöhnlicher Temperatur zu, so erhält man eine Fällung erst mit dem achten Cubikcentimeter. Unter diesen Umständen scheint also das Silberchromat eine dreimal so grosse Löslichkeit zu haben; das Gleichgewicht stellt sich ungefähr der folgenden Formel entsprechend:

$$
3 \mathrm{~K}_{2} \mathrm{CrO} \mathrm{O}_{4}+6 \mathrm{AgNO}_{3}=2 \mathrm{~K}_{2} \mathrm{CrO}_{4}+\mathrm{Ag}_{2} \mathrm{CrO}
$$$$
+2 \mathrm{~K} \mathrm{NO}_{3}+4 \mathrm{AgNO}_{3} \text {. }
$$

Die Zufügung zu einer solchen Auflösung, sei es von Kaliumchromat oder Silbernitrat, muss nothwendig, indem sie das Verhältniss des Silberchromats vermehrt, die Fällung dieses Salzes bestimmen. So wäre das Bedürfoiss einer nicht zu geringen Menge Chromat, als Indicator im schon erwähnten Mohr'schen Verfahreu anzuwenden, erklärlich.

3. Bestimmung der Chloride. Das Verfabren besteht darin, die Auflösung des Chlorids mit einem Übermaass von Silberchromat zu behandeln, dann zu filtriren und in dem Filtrate das gebildete lösliche Chromat jodometrisch zu bestimmen. Die Reihenfolge der Umsetzungen wird durch folgende Formeln ausgedrückt:

A. $4 \mathrm{NaCl}+\mathrm{x} \mathrm{Ag}_{2} \mathrm{CrO}_{4}=2 \mathrm{Na}_{2} \mathrm{CrO}_{4}+4 \mathrm{Ag} \mathrm{Cl}$ $+(\mathrm{x}-2) \mathrm{Ag}_{2} \mathrm{Cr} \mathrm{O}$.

B. $2 \mathrm{Na}_{2} \mathrm{CrO}_{4}+12 \mathrm{KJ}+19 \mathrm{H}_{2} \mathrm{SO}_{4}=4 \mathrm{NaHSO}_{4}$ $+12 \mathrm{KH} \mathrm{SO}_{4}+\mathrm{Cr}_{2}\left(\mathrm{SO}_{4}\right)_{3}+22 \mathrm{H}_{2} \mathrm{O}+12 \mathrm{~J}$.

C. $12 \mathrm{~J}+12 \mathrm{Na}_{2} \mathrm{~S}_{2} \mathrm{O}_{3}=12 \mathrm{NaCl}+6 \mathrm{Na}_{2} \mathrm{~S}_{4} \mathrm{O}_{6}$.

Daraus geht hervor, dass 1 Chloratom 3 Jodatome, also $3 \mathrm{Mol}$. Natriumbyposulfit, entsprechen.

Unsere ersten Versuche waren, mit reinem Chlornatrium und ohne der Löslichkeit des Silberchromats Rechnung zu tragen, ausgeführt. Die Resultate waren folgende:

\begin{tabular}{c|c|c}
\hline $\begin{array}{c}\text { Na CI } \\
\text { angewandt } \\
\mathbf{m g}\end{array}$ & $\begin{array}{c}\text { Na Gi } \\
\text { gefunden } \\
\text { mg }\end{array}$ & $\begin{array}{c}\text { Differenz } \\
\mathbf{m g}\end{array}$ \\
\hline 144,5 & 145,2 & $+0,7$ \\
98,0 & 98,5 & $+0,5$ \\
88,5 & 89,2 & $+0,7$ \\
72,9 & 73,2 & $+0,3$
\end{tabular}

In einer anderen Versuchsreihe haben wir die Löslichkeit des Silberchromats in Wasser beachtet, indem wir jedesmal zwei parallele Analysen machten, wovon eine ohne Chlorid.

Bevor man das Silberchromat dem Chlorid zufügte, wurde es durch Schütteln in dem Wasser suspendirt; dann entuahm man von der trüben Flüssigkeit eine gewisse Anzahl Cubikcentimeter vermittels einer Pipette, so dass man einen ziemlich starken Uberschuss an Silberchromat hatte. Der Niederschlag wurde jedesmal auf die nämliche Art und mit derselben Wassermenge gewaschen. In beiden Fällen wurde dieselbe Anzahl Cubikcentimeter Jodkalium und verdünnter Schwefelsäure zugefügt. Endlich wurde das, in der ersten Probe angewendete Volumen an unterschwefligsaurem Natron, von dem in der zweiten Probe gebrauchten substrahirt. Dieses Volumen könnte ein für alle Mal bestimmt werden, wenn die zu bestimmenden Mengen Chlor nur verhältnissmässig wenig von einander verschieden sein sollten.

Dieses Verfahren hat einen doppelten Vortheil: Wenn, was wohl vorkommen kann, das angewandte Silberchromat mit ein wenig Silberdichromat verunreinigt sein sollte, so sind, da man jedesmal dieselben Mengen des Reagens entnimmt, die in dem Filtrate gefundenen Silberdichromatmengen dieselben und der Fehler wird ausgeglichen. Das für die Bestimmung angewandte Jodkalium kann andererseits Jodate enthalten; das besprochene Verfahren gleicht ebenfalls den hieraus entstehenden Fehler aus.

Unter diesen Umständen wurden folgende Resultate erzielt:

\begin{tabular}{c|c|c}
\hline $\begin{array}{c}\mathrm{Na} \mathrm{Cl} \\
\text { angewandt } \\
\mathbf{m g}\end{array}$ & $\begin{array}{c}\text { Na Cl } \\
\text { gefunden } \\
\mathbf{m g}\end{array}$ & $\begin{array}{c}\text { Differenz } \\
\mathbf{m g}\end{array}$ \\
\hline 27,6 & 27,4 & $-0,2$ \\
4,98 & 4,94 & $-0,04$ \\
1,245 & 1,210 & $-0,035$
\end{tabular}

Das Verfahren kann man vereinfachen, ohne der Genauigkeit der Resultate zu schaden, im Gegentheil, indem man die Reaction in einem Messkolben stattfinden lässt und man das gebildete Chromat nur in einem aliquoten Theile der filtrirten oder durch Absetzen völlig geklärten Flüssigkeit bestimmt. Da Silberchromat in einer verdünnten Chromatauflösung nicht löslich ist, so braucht man dessen Löslichkeit in Wasser, wie es bei vollständiger Filtration und Waschen des Rückstandes der Fall ist, nicht weiter zu beachten.

Folgende unter diesen Umständen ausgeführte Versuche ergaben:

\begin{tabular}{c|c|c}
\hline $\begin{array}{c}\text { Na Cl } \\
\text { angewandt } \\
\text { mg }\end{array}$ & $\begin{array}{c}\text { Na Cl } \\
\text { gefunden } \\
\mathbf{m g}\end{array}$ & $\begin{array}{c}\text { Differenz } \\
\mathbf{m g}\end{array}$ \\
\hline 100 & 99,7 & $-0,3$ \\
100 & 100,2 & $+0,2$ \\
$\mathbf{4 0}$ & 39,82 & $-0,18$ \\
20 & 19,91 & $-0,09$ \\
20 & 20,01 & $+0,01$ \\
10 & 10,4 & $+0,4$
\end{tabular}


Was die Vorsichten angeht, welche man in der Anwendung der Methode beobachten muss, ist es im Allgemeinen empfehlenswerth :

1. absolut reines Silberchromat unter Wasser und im Dunkeln auf bewahrt zu gebrauchen;

2. nur in durchaus neutralen Auflösungen zu operiren, denn das Silberchromat ist nur unter solchen Bedingungen beständig. Ist die Auflösung elwas sauer, so kann man dieselbe mit kohlensaurem. Kalk neutralisiren, den man durch Fällung von salpetersaurem Kalk durch kohlensaures Natron erhält, indem man das Nitrat vorherrschen lässt; der Niederschlag ist durch Decantation zu waschen und unter Wasser aufzubewahren. Wenn man dagegen mit einer alkalischen Auflösung zu thun hat, so muss man mit verdünnter Salpetersäure neutralisiren, wovon nachher, wenn nöthig, ein Übermaass ebenfalls mit kohlensaurem Kalk neutralisirt werden kann;

3. man muss selbstredend die für die jodometrischen Bestimmungen des Chromats bekannten Vorsichtsmaassregeln treffen.

Endlich fügen wir noch zu, dass die Anwesenheit sehr grosser Mengen von schwefelsauren Alkalien Fehler verursachen können.

Um die Operationen noch zu verkürzen, könnte man für äusserst geringe Mengen Chloride das durch doppelten Austausch gebildete Chromat colorimetrisch bestimmen.

4. Bestimmung der Bromide und Jodide. Die Bestimmung der Bromide und Jodide kann vollständig wie die der Chloride ausgeführt werden. Nur ist zu bemerken, dass das Silberbromid oder Jodid oft mit dem löslichen Chromat durch das Filter geht; aber diese Thatsache, die in unseren Versuchen mit Natriumchlorid nicht stattgefunden hat, verbindert die Operationen der Titration des Chromats nicht. Folgende Versuche fanden mit Kaliumbromid statt :

\begin{tabular}{c|c|c}
\hline $\begin{array}{c}\mathbf{K ~ B r} \\
\text { algew andt } \\
\mathbf{m g}\end{array}$ & $\begin{array}{c}\mathbf{K ~ B r} \\
\text { gefunden } \\
\mathbf{m g}\end{array}$ & $\begin{array}{c}\text { Differenz } \\
\mathbf{m g}\end{array}$ \\
\hline $\mathbf{1 6 , 7}$ & 16,8 & $+0,1$ \\
$\mathbf{3 3 , 4}$ & 33,7 & $+0,3$ \\
50,1 & 50,6 & $+0,5$ \\
$\mathbf{8 3 , 5}$ & 84,1 & $+0,6$
\end{tabular}

Folgende Resultate erhielten wir endlich mit Kaliumjodid:

\begin{tabular}{c|c|c}
\hline $\begin{array}{c}\mathbf{K} \mathbf{J} \\
\text { angewandt } \\
\mathbf{m g}\end{array}$ & $\begin{array}{c}\mathbf{K ~ J} \\
\text { gefunden } \\
\mathbf{m g}\end{array}$ & $\begin{array}{c}\text { Differenz } \\
\mathbf{m g}\end{array}$ \\
\hline 20 & 20,1 & $+0,1$ \\
60 & $\mathbf{5 9 , 7}$ & $\mathbf{- 0 , 3}$ \\
100 & $\mathbf{1 0 0 , 4}$ & $+0,4$
\end{tabular}

Aus dem Vorhergehenden kann man also schliessen, dass die Methode ebenso wohl für Bromide und Jodide als für Chloride gilt.
Sowohl für die einen als auch für die anderen fehlt nichts, was Genauigkeit angeht. Für gewöhnliche Bestimmungen und namentlich wenn die zu bestimmende Quantität ziemlich bedeutend ist, kann sie dem einfacheren und bequemen Verfahren von Gay Lussac und Mohr selbstredend nicht entgegen treten; für genaue Bestimmung von geringeren Mengen jedoch, wie z. B. im Trinkwasser, erscheint sie uns gute Dienste leisten zu können.

Laboratorium der analytischen Chemie der Universität Lüttich. Januar 1891.

\section{Denaturirter Spiritus.}

In dieser Z. 1888, Heft 22 habe ich Mittheilungen über denaturirten Spiritus gemacht, welcher unter Anderem Messinglampen angriff und dadurch Unzuträglichkeiten, namentlich Zerstörung der Platintiegel beim Glühen hervorrief. Julius Schenkel (d. Z. $1889,66)$ bemerkt, dass bis zum Juli 1888 der Geruch des denaturirten Spiritus seitens vieler Händler durch Zusatz von Säuren und ätherischen Ölen "verbessert" wurde, wodurch die Zerstörung der Messinglampen herbeigeführt werden konnte. Durch Bundesrathsbeschluss rom 21. Juni 1888 sei jeder Zusatz zum denaturirten Spiritus verboten, durch welchen das Denaturirungsmittel ganz oder theilweise ausgeschieden wird. Zum Schluss sagt Schenkel: "Der angesäuerte Spiritus ist jetzt aus dem Handel verschwunden, es sind demnach obige Klagen über den denaturirten Spiritus hinfällig geworden".

Diese meine Klagen über den denaturirten Spiritus sind indessen noch nicht hinfällig geworden, denn im December 1890 bezog ich ein Fass ( 1 hl) denaturirten Spiritus aus Beuthen, O.-Schl., welcher zum mindesten die Messinglampen ebenso rasch zerstörte und die Platintiegel in kurzer Zeit ruinirte, wie derjenige denaturirte Spiritus, über den ich seiner Zeit die ebenso berechtigte Klage geführt habe. Ich glaube weniger an einen absichtlichen Säurezusatz, als vielmehr an eine unabsichtliche Verwechslung von Holzgeist mit Holzessig. Dass eine derartige Verwechslung nicht unmöglich ist, erhellt daraus, dass mir in einer Droguenhandlung statt Holzgeist thatsächlich Holzessig verkauft wurde.

C. Reinhardt. 